\title{
Pneumococcal Meningitis in Adults after Introduction of PCV7 and PCV13, Israel, July 2009-June 2015
}

\author{
Gili Regev-Yochay, Klaris Reisenberg, Michal Katzir, Yonit Wiener-Well, Galia Rahav, \\ Jacob Strahilevitz, Valery Istomin, Evgenia Tsyba, Avi Peretz, Shirley Khakshoor, \\ Ron Dagan, on behalf of the Israeli Adult Invasive Pneumococcal Disease Group ${ }^{2}$
}

\begin{abstract}
The indirect effect of pneumococcal conjugate vaccine on adult pneumococcal meningitis has not been thoroughly investigated. We present data from active surveillance on pneumococcal meningitis in adults in Israel occurring during July 2009-June 2015. Pneumococcal meningitis was diagnosed for 221 patients, $9.4 \%$ of all invasive pneumococcal disease (IPD) cases. Although overall IPD incidence decreased during the study period, meningitis increased nonsignificantly from 0.66 to 0.85 cases $/ 100,000$ population. Incidence of vaccine type (VT) pneumococcal meningitis (VT13) decreased by $70 \%$, but non-VT13 pneumococcal meningitis increased from 0.32 to 0.75 cases $/ 100,000$ population (incident rate ratio $2.35,95 \% \mathrm{Cl} 1.27-4.35$ ). Pneumococcal meningitis patients were younger and healthier than nonmeningitis IPD patients, and $20.2 \%$ had a history of previous head surgery or cerebrospinal fluid leak compared with $<2.0 \%$ of nonmeningitis patients $(p<0.0001)$. Non-VT13 types that rarely cause IPD $(15 B / C, 6 C, 23 A, 23 B, 24 F)$ seem to be emerging as common causes of meningitis.
\end{abstract}

Ctreptococcus pneumoniae is the leading cause of bacte$\mathcal{N}$ rial meningitis for persons of all ages (1). Pneumococcal meningitis is a relatively rare but the most severe form

Author affiliations: Sheba Medical Center, Ramat Gan, Israel (G. Regev-Yochay, G. Rahav, S. Khakshoor); Tel Aviv University, Tel Aviv, Israel (G. Regev-Yochay, M. Katzir, G. Rahav); Soroka Medical Center, Beersheba, Israel (K. Reisenberg); Bar-llan University, Safed, Israel (K. Reisenberg, A. Peretz); Meir Medical Center, Kfar Saba, Israel (M. Katzir); Shaare Zedek Medical Center, Jerusalem, Israel (Y. Wiener-Well); HadassahHebrew University, Jerusalem (J. Strahilevitz); Hillel Yaffe Medical Center, Hadera, Israel (V. Istomin); Barzilai Medical Center, Ashkelon, Israel (E. Tsyba); Baruch Padeh Medical Center, Tiberias, Israel (A. Peretz); Ben-Gurion University of the Negev, Beersheba (R. Dagan)

DOI: https://doi.org/10.3201/eid2407.170721 of invasive pneumococcal disease (IPD). Untreated pneumococcal meningitis usually leads to death, and even with optimal treatment, mortality rates are high and disease is severe with frequent long-term sequelae $(1,2)$.

Since the introduction of pneumococcal conjugate vaccines (PCVs) into the national immunization plans (NIPs) for children in different countries, IPD incidence has declined, not only among children but also among unvaccinated adult populations through herd (indirect) protection (3-6). Despite non-VT strains nearly completely replacing VT strains as the causative agents of invasive nasopharynx disease, this replacement by non-VT strains was only partial in both the pediatric and adult populations, presumably because of the lower invasive potential of most non-VT strains (7). However, a higher magnitude replacement of the serotypes associated with invasive disease was observed among immunocompromised and elderly populations than among the rest of the general population $(4,8)$. Although several studies have assessed the effect of PCV on meningitis in children $(9-11)$, data on the indirect effects on adult pneumococcal meningitis are scarce. The available data mostly address the effect of the 7-valent PCV (PCV7) $(12,13)$ or PCV10 on pneumococcal meningitis in adults (14), and only 2 studies addressed the effect of PCV13 $(15,16)$.

In Israel, pneumococcal polysaccharide vaccine 23 (PPSV23) has been part of the NIP for adults for many years; the constant coverage rate is $>70 \%$ for adults $\geq 65$ years of age. Administration of PCV7 at 2, 4, and 12 months of age and a catch-up plan was introduced to the NIP for children in July 2009 , resulting in $>70 \%$ vaccine

\footnotetext{
${ }^{1}$ Some results from this study were presented at the 10th International Symposium on Pneumococci and Pneumococcal Diseases, June 26-30, 2016, Glasgow, Scotland.
}

${ }^{2}$ Additional members of the Israeli Adult Invasive Pneumococcal Disease Group are listed at the end of this article. 
coverage ( $\geq 2$ doses) for children $<2$ years of age within 1 year of implementation (17). Starting in October 2010, administration of PCV13 began to replace that of PCV7, and immunization coverage rapidly reached $\approx 95 \%$ for 2 doses and $\approx 90 \%$ for 3 doses (18). We previously described the indirect effect of PCV on the adult population in Israel and reported an $\approx 20 \%$ decrease of overall IPD incidence 4 years after the introduction of PCV7 and 2.5 years after the introduction of PCV13; we also reported that the proportion of pneumococcal meningitis cases among all IPD cases increased and that incidence did not decrease as did other IPDs (4). In this article, we assess meningitis IPD and nonmeningitis IPD incidence and the change in associated serotypes in adults 6 years after PCV7 introduction.

\section{Materials and Methods}

\section{Ethics Statement}

This study was conducted after protocols were approved by the Sheba Medical Center Institutional Review Board (Ramat Gan, Israel) and the Soroka University Medical Center Institutional Review Board (Beersheba, Israel). Because this study was a retrospective observational study, the institutional review boards waived the need for written informed consent, so informed consent was not obtained from participants. Therefore, all patient records and information were deidentified before analysis.

\section{Study Period and Population}

The period evaluated was July 1, 2009-June 30, 2015, a 6-year period starting when PCV7 was introduced into the NIP for children. We included all culture-confirmed IPD patients of the adult ( $\geq 18$ years of age) population in Israel ( $\mathrm{n}=5,029,600$ in $2009 ; \mathrm{n}=5,504,900$ in 2015; http://www. cbs.gov.il).

\section{Surveillance System and Study Design}

To ensure both a high rate of reporting and data collection from the medical records, a large research network named the Israeli Adult Invasive Pneumococcal Disease (IAIPD) group was established. This group includes 2 researchers from each of the 27 acute care hospitals of Israel: a researcher from the microbiology laboratory and an infectious disease physician devoted to following the IPD patients and collecting the required data, as described previously (4).

In Israel, all invasive $S$. pneumoniae isolates are required by law to be reported and sent to the Ministry of Health reference laboratory (Jerusalem, Israel), and several different methods were used by clinical staff members to collect these bacterial isolates before submission. In addition to this passive surveillance, active surveillance was performed involving a capture-recapture method, in which the IAIPD representative at each of the 27 laboratories performing blood and cerebrospinal fluid (CSF) cultures reported all invasive $S$. pneumoniae isolates on a weekly basis and transported them to study headquarters (Pediatric Infectious Disease Laboratory, Soroka University Medical Center), as described previously (18).

IAIPD investigators retrospectively collected data for every laboratory-identified case from the medical files. Data were available from 24 of the 27 centers, constituting $90.9 \%$ of all IPD cases that were identified. Data collected included sociodemographic data (sex, age, place of birth, city of residence); medical history, including concurrent medical conditions and IPD-predisposing medical conditions; substance abuse; smoking history; influenza in the days preceding hospitalization; and vaccination history for influenza and pneumococcal pneumonia. The Centers for Disease Control and Prevention definition for IPD-predisposing medical conditions, on which vaccination recommendations are based (https://www.cdc.gov/vaccines/vpd/ pneumo/downloads/pneumo-vaccine-timing.pdf), was used to divide adults into 3 categories: 1) high-risk patients, defined as patients having chronic renal failure, HIV, medically induced or innate immunodeficiencies, asplenia, hematologic or metastatic malignancies, CSF leak, or prior neurosurgery; 2) at-risk patients, defined as patients with diabetes mellitus, congestive heart failure, chronic lung disease, cirrhosis, or an addiction to alcohol; and 3) not atrisk patients, defined as those who were not recognized as having a predisposing medical condition. According to the NIP for adults in Israel, it is recommended that high-risk patients be vaccinated with PCV13 and PPSV23 and atrisk patients with PPSV23. We also collected data on inhospital complications: septic shock, need for ventilation, disability as determined by transition to a long-term care facility, and concurrent medical conditions.

\section{Case Definition}

We defined IPD cases on the basis of positive pneumococcal cultures and did not include PCR testing or antigen detection, as described previously (4). We defined pneumococcal meningitis patients as those with an $S$. pneumoniae-positive CSF culture or those with an S. pneumoniae-positive blood culture who were also given a clinical diagnosis of meningitis by the treating physician (i.e., given a discharge diagnosis code of meningitis).

\section{Vaccination Policy and Vaccine Uptake}

PCV7 was licensed in Israel in 2007 and was introduced into the NIP for children in July 2009 with a 2-, 4-, and 12-month schedule and a 2-dose catch-up plan for all children $<2$ years of age at the time of introduction. The methods used to evaluate vaccine uptake initiated in July 2009 are described elsewhere (18). 


\section{Laboratory Testing}

Susceptibility testing of isolates was performed at the local laboratory of each medical center. All centers assessed susceptibility to penicillin and ceftriaxone, including MIC determination by using ETEST (bioMérieux, Marcy l'Etoile, France), following the Clinical and Laboratory Standards Institute guidelines (http://www.facm.ucl.ac.be/intranet/ CLSI/CLSI-2017-M100-S27.pdf). Serotyping was performed with all viable isolates at the headquarters laboratory by using the Quellung reaction (Staten Serum Institute, Copenhagen, Denmark).

\section{Statistical Analyses}

We determined the denominators for calculating the incidence and mortality rates by using data from the Israeli Central Bureau of Statistics (http://www.cbs.gov. il). We calculated incidence rate ratios (IRRs) by dividing the incidence rate (IR) of year 6 by the IR of year 1. We calculated $95 \%$ Poisson CIs for IRs and IRRs by following the method of Greenland and Rothman (19). We performed a Poisson regression to test the trend in IRs over the 6-year study. We used multivariate logistic models to determine the predictors of death among IPD patients and the predictors of meningitis development among meningitis patients who were not at risk for IPD. In the pneumococcal meningitis mortality rate model, we included the following variables: age, sex, predisposing medical conditions, nonhematologic metastatic malignancies, smoking history, and source of infection. In the models tested to define predictors of meningitis development among not at-risk patients, we included the following variables: age, concurrent medical conditions (other than those known to be predisposing for IPD), and serotype. We grouped serotypes by vaccine coverage in 1 model and specifically tested serogroup 23 in another model. We presented the model with the higher goodness-of-fit (model with serogroup 23 serving as the predictor). We included predictor variables with $\mathrm{p}$ values $<0.2$ in the univariate analysis in the respective multivariate model. We calculated adjusted odds ratios, 95\% CIs, and adjusted $\mathrm{p}$ values and used SAS 9.4 software (SAS Institute, Cary, NC, USA). We calculated the Simpson diversity index to assess the change in diversity of serotypes in the bacterial population (20).

\section{Results}

During the 6-year study period, 2,579 IPD cases occurred, but 234 were excluded because of a lack of medical file data; 221 pneumococcal meningitis diagnoses in persons $\geq 18$ years of age were reported, constituting $9.4 \%$ of all the IPD cases with diagnosis data available $(\mathrm{n}=2,345)$. Of the 221 patients with pneumococcal meningitis diagnoses, S. pneumoniae was isolated from both CSF and blood cultures in $99(44.8 \%)$ episodes, blood cultures only in 89 (40.3\%), and CSF only in $33(14.9 \%)$.

The mean annual incidence of pneumococcal meningitis was 0.77 cases $/ 100,000$ population, rising from 0.66 cases/100,000 population in the first year to 0.85 cases/100,000 population in the last study year, representing a $29 \%$ nonsignificant increase (IRR $1.29,95 \%$ CI $0.81-2.06$ ) (Figure 1; online Technical Appendix Table 1, https://wwwnc.cdc.gov/EID/article/24/7/17-0721-Techapp1.pdf). Over the same period, the incidence of nonmeningitis IPD decreased significantly by $26.7 \%$, from 8.49 cases $/ 100,000$ population to 6.22 cases $/ 100,000$ population (IRR 0.73, 95\% CI 0.63-0.85).

\section{Characteristics of Pneumococcal Meningitis Patients}

Similar to other IPD patients, $75 \%$ of meningitis IPD patients were of Jewish ethnicity, and $\approx 50 \%$ were men (similar to the distribution in the general population) (Table 1). Patients with pneumococcal meningitis were significantly younger than IPD patients without meningitis $(\mathrm{p}<0.0001)$. Moreover, concurrent medical conditions were less frequent among the meningitis IPD patients than the nonmeningitis IPD patients; $87.3 \%$ of meningitis patients and $92.8 \%$ of nonmeningitis patients $(\mathrm{p}=0.004)$ had any concurrent medical condition, and $58.7 \%$ of meningitis patients and $68.6 \%$ of nonmeningitis patients $(\mathrm{p}=0.003)$ were at risk or at high risk for IPD (had concurrent medical conditions for which PPSV23 is recommended). The most common high-risk concurrent medical condition among the pneumococcal meningitis patients, occurring in $20.2 \%$ of the population, was previous neurosurgery or CSF leak, which only occurred in $2.0 \%$ of the nonmeningitis IPD patients $(\mathrm{p}<0.0001)$.

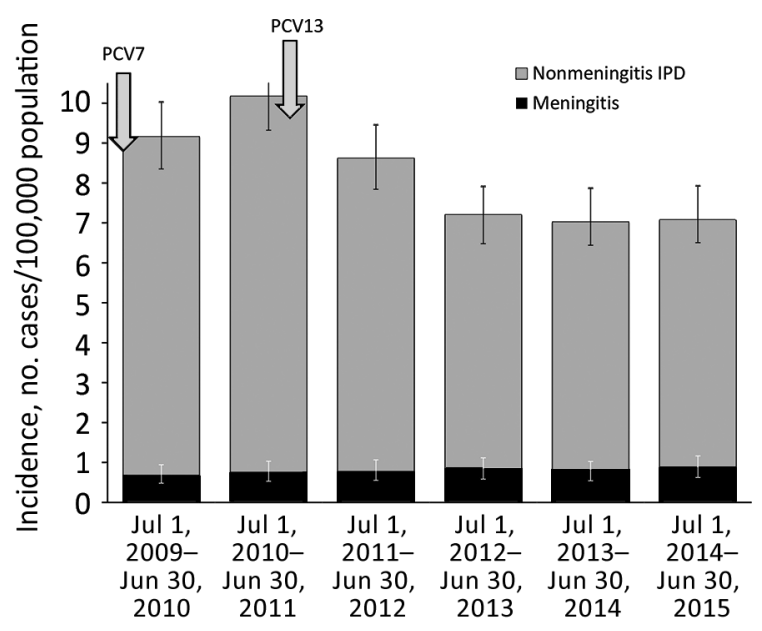

Figure 1. Incidence of meningitis and nonmeningitis IPD in patients $\geq 18$ years of age, Israel, July 1, 2009-June 30, 2015. Introduction of PCV7 and PCV13 into the pediatric national immunization plan is depicted with arrows; 95\% Poisson Cls are depicted for overall IPD and meningitis IPD. IPD, invasive pneumococcal disease; PCV, pneumococcal conjugate vaccine. 
Table 1. Univariate analysis of the characteristics of patients with meningitis IPD and nonmeningitis IPD by IPD source, Israel, July 1 , 2009-June 30, 2015*

\begin{tabular}{|c|c|c|c|c|c|c|}
\hline \multirow[b]{2}{*}{ Variable } & \multirow[b]{2}{*}{$\begin{array}{c}\text { Meningitis } \\
\text { IPD, } n=221\end{array}$} & \multicolumn{4}{|c|}{ Nonmeningitis IPD, $\mathrm{n}=2,124 \dagger$} & \multirow[b]{2}{*}{$p$ value§ } \\
\hline & & Pneumonia & $\begin{array}{c}\text { Bacteremia, } \\
\text { no source }\end{array}$ & $\begin{array}{c}\text { IPD, rare } \\
\text { types } \ddagger\end{array}$ & All & \\
\hline Population with full data available & 213 & 1,596 & 301 & 203 & 2,124 & \\
\hline \multicolumn{7}{|l|}{ Sex\| } \\
\hline M & $104(48.6)$ & $890(55.8)$ & $171(56.8)$ & $108(53.2)$ & $1,182(55.7)$ & 0.047 \\
\hline $\mathrm{F}$ & $110(51.4)$ & $704(44.2)$ & $130(43.2)$ & $95(46.8)$ & $940(44.3)$ & \\
\hline Jewish ethnicity & $166(75.5)$ & $1,157(72.6)$ & $221(73.7)$ & $154(75.9)$ & $1,551(73.0)$ & 0.46 \\
\hline Age, yள & & & & & & $<0.0001$ \\
\hline Mean & 57.68 & 64.83 & 61.83 & 64.03 & 64.37 & \\
\hline Median (range) & 61.47 & 66.53 & 65.45 & 66.91 & 66.35 & \\
\hline & $(18.2-91.3)$ & $(18.1-105.0)$ & $(18.1-98.3)$ & $(18.1-97.8)$ & $(18.1-105.0)$ & \\
\hline $18-49$ & $72(32.6)$ & $368(23.1)$ & $74(24.6)$ & $45(22.2)$ & $491(23.1)$ & \\
\hline $50-64$ & $68(30.8)$ & $382(23.9)$ & $75(24.9)$ & $47(23.2)$ & $510(24.0)$ & \\
\hline$\geq 65$ & $81(36.7)$ & $846(53.0)$ & $152(50.5)$ & $111(54.7)$ & $1,123(52.9)$ & \\
\hline \multicolumn{7}{|l|}{ Concurrent medical conditions } \\
\hline Any & $186(87.3)$ & $1,481(92.8)$ & $287(95.4)$ & $189(93.1)$ & $1,972(92.8)$ & 0.004 \\
\hline High risk and at risk & $130(61.0)$ & $1,093(68.5)$ & $233(77.4)$ & $156(76.49)$ & $1,495(70.4)$ & 0.005 \\
\hline High risk & $81(38.0)$ & $645(40.4)$ & $179(59.5)$ & $107(52.7)$ & $939(44.2)$ & 0.083 \\
\hline Any immunosuppression & $16(7.5)$ & $187(11.7)$ & $61(20.3)$ & $35(17.2)$ & $285(13.4)$ & 0.014 \\
\hline Bone marrow transplantation & $4(1.9)$ & $42(2.6)$ & $17(5.7)$ & $12(5.9)$ & $71(3.3)$ & 0.248 \\
\hline HIV & 0 & $33(2.1)$ & $3(1.0)$ & $3(1.5)$ & $39(1.8)$ & 0.046 \\
\hline Hematologic malignancy & $19(8.9)$ & $208(13.0)$ & $68(22.6)$ & $41(20.2)$ & $320(15.1)$ & 0.015 \\
\hline Metastatic cancer & $3(1.4)$ & $73(4.6)$ & $34(11.3)$ & $8(3.9)$ & $116(5.5)$ & 0.010 \\
\hline Asplenia & $9(4.2)$ & $26(1.6)$ & $18(6.0)$ & $14(6.9)$ & $58(2.7)$ & 0.213 \\
\hline Chronic renal failure & $14(6.6)$ & $277(17.4)$ & $46(15.3)$ & $35(17.2)$ & $363(17.1)$ & $<0.0001$ \\
\hline Previous neurosurgery, CSF leak & $43(20.2)$ & $29(1.8)$ & $6(2.0)$ & $7(3.5)$ & $42(2.0)$ & $<0.0001$ \\
\hline At risk & 49 (23.0) & $448(28.1)$ & $54(17.9)$ & $49(24.1)$ & $556(26.2)$ & 0.314 \\
\hline Congestive heart failure & $13(6.1)$ & $270(16.9)$ & $38(12.6)$ & $36(17.7)$ & $350(16.5)$ & $<0.0001$ \\
\hline Chronic lung disease & $20(9.4)$ & $358(22.5)$ & $35(11.6)$ & $29(14.3)$ & $425(20.0)$ & 0.0002 \\
\hline Cirrhosis & $2(0.9)$ & $29(1.8)$ & $16(5.3)$ & $17(8.4)$ & $62(2.9)$ & 0.091 \\
\hline Diabetes mellitus & $47(22.1)$ & $437(27.4)$ & 87 (28.9) & $54(26.6)$ & $584(27.5)$ & 0.089 \\
\hline Alcohol abuse & $3(1.4)$ & $51(3.2)$ & $10(3.3)$ & $8(3.9)$ & $69(3.3)$ & 0.138 \\
\hline Healthy & $27(12.7)$ & $115(7.2)$ & $14(4.7)$ & $14(6.9)$ & $152(7.2)$ & 0.004 \\
\hline \multicolumn{7}{|c|}{$\begin{array}{l}\text { *Values are no. (\%) except as indicated. CSF, cerebospinal fluid; IPD, invasive pneumococcal disease. } \\
\text { †The source of } 24 \text { nonmeningitis IPD cases was not reported, so these cases were only included in the "All" column. } \\
\text { tThese IPD cases occurred after endocarditis, sinusitis, mastoiditis, peritonitis, endometritis, osteomyelitis, cellulitis, or septic arthritis; a few resulted from } \\
\text { abscesses in various locations. } \\
\text { \$p value of meningitis vs. nonmeningitis IPD cases. Bold indicates significance. } \\
\text { TThe denominators are the total number of cases with information available pertaining to the relevant variable. }\end{array}$} \\
\hline
\end{tabular}

\section{Outcomes}

Only in-hospital outcomes were available. The crude overall case-fatality rate was $15.5 \%$ for meningitis IPD patients and $23.0 \%$ for nonmeningitis IPD patients $(\mathrm{p}=$ 0.0123) (Table 2). After adjusting for age, risk group, and VT in a multivariate logistic model, we found that the mortality rate of patients with pneumococcal meningitis did not differ from that of patients with nonmeningitis IPD (Table 3). Other outcomes were worse for pneumococcal meningitis patients; compared with nonmeningitis IPD patients, meningitis IPD patients were more frequently hospitalized in the intensive care unit, more frequently required mechanical ventilation, and had longer lengths of hospital stay (median 6 days for nonmeningitis IPD vs. 15 days for meningitis IPD; Table 2 ). In addition, nearly $20 \%$ of meningitis IPD patients were discharged to long-term care facilities compared with $7 \%$ of nonmeningitis IPD patients.

\section{Antimicrobial Drug Resistance}

Among the 221 isolates from meningitis IPD patients, 211 were susceptible to penicillin and 180 were susceptible to ceftriaxone. Isolates resistant to penicillin (MIC $>0.06 \mu \mathrm{g} /$ $\mathrm{mL}$ ) were isolated from $25.1 \%$ of all meningitis patients. Ceftriaxone nonsusceptibility ( $\mathrm{MIC} \geq 1 \mu \mathrm{g} / \mathrm{mL}$ ) emerged during the study years, from $2.1 \%(\mathrm{n}=1)$ in the first 2 years to $>8 \%(n=6)$ in the last 2 years (Figure 2$)$. The single ceftriaxone-nonsusceptible meningitis isolate found during the first 2 years was serotype 14, but in the last 2 years, most ceftriaxone-nonsusceptible isolates were serotypes 19A and $23 \mathrm{~F}$. A single isolate of serotype $23 \mathrm{~F}$ was highly resistant to ceftriaxone (MIC $2 \mu \mathrm{g} / \mathrm{mL}$ ) and penicillin (MIC $8 \mu \mathrm{g} / \mathrm{mL}$ ). The proportion of isolates from nonmeningitis IPD patients resistant to ceftriaxone, with a $\mathrm{MIC} \geq 1 \mu \mathrm{g} / \mathrm{mL}(\approx 4 \%)$, did not change over the course of the study. Serotypes 14 and $19 \mathrm{~A}$ each accounted for nearly $30 \%$ of the isolates, and $19 \mathrm{~F}$ for $12 \%$. All isolates were susceptible to vancomycin. 
Table 2. Univariate analysis of clinical outcomes of meningitis versus nonmeningitis IPD patients, Israel, July 1, 2009-June 30, $\underline{2015^{*}}$

\begin{tabular}{|c|c|c|c|}
\hline Category & $\begin{array}{c}\text { Pneumococcal } \\
\text { meningitis, } \\
n=221\end{array}$ & $\begin{array}{l}\text { Nonmeningitis } \\
\text { IPD, } n=2,124\end{array}$ & $p$ value \\
\hline $\begin{array}{l}\text { Population with full } \\
\text { data available }\end{array}$ & 213 & 2,123 & \\
\hline $\begin{array}{l}\text { Overall no. deaths } \\
\text { (case-fatality rate) }\end{array}$ & $33(15.5)$ & $488(23.0)$ & 0.0123 \\
\hline ICU admission & $115(54.0)$ & $322(15.2)$ & $<0.0001$ \\
\hline Ventilation required & $91(42.7)$ & 405 (19.1) & $<0.0001$ \\
\hline Mean LOS, d $\dagger$ & 21.0 & 10.5 & $<0.0001$ \\
\hline \multicolumn{4}{|c|}{ Percentile LOS, by days, $\%$} \\
\hline 5 & 55 & 33 & \\
\hline 10 & 38.5 & 21 & \\
\hline 25 & 21 & 11 & \\
\hline 50 & 15 & 6 & \\
\hline 75 & 12 & 4 & \\
\hline 90 & 10 & 2 & \\
\hline Sepsis $†$ & $10 / 180(5.6)$ & $170 / 1,635(10.4)$ & 0.0391 \\
\hline $\begin{array}{l}\text { Discharged to long- } \\
\text { term care facility } \dagger\end{array}$ & $34 / 173(19.7)$ & $104 / 1,490(7.0)$ & $<0.0001$ \\
\hline \multicolumn{4}{|c|}{$\begin{array}{l}\text { *Values are no. (\%) except as indicated. ICU, intensive care unit; IPD, } \\
\text { invasive pneumococcal disease; LOS, length of stay. } \\
\text { †Outcome assessed only for those who were discharged (deaths were } \\
\text { excluded). }\end{array}$} \\
\hline
\end{tabular}

\section{Serotype Dynamics}

Although overall pneumococcal meningitis incidence did not decrease, meningitis caused by VT13 serotypes decreased by $70 \%$ (IRR $0.297,95 \%$ CI $0.11-0.82$ ), similar to the decrease in VT13 nonmeningitis IPD cases. VT7 serotypes were totally eliminated by the sixth study year (Figure 3 ). The PCV13 serotypes 3, 7F, and 19A caused meningitis in the sixth study year and constituted $11.9 \%$ of all isolates

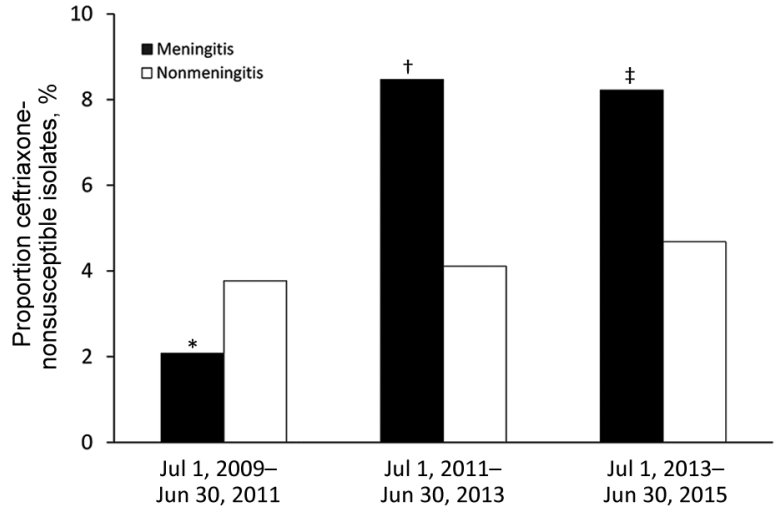

Figure 2. Proportion ceftriaxone-nonsusceptible isolates among all Streptococcus pneumoniae isolates acquired from patients with invasive pneumococcal disease, by 2-year period, Israel, July 1, 2009-June 30, 2015. Ceftriaxone-nonsusceptible isolates were those that could grow at a concentration above ceftriaxone's MIC ( $\geq 1 \mu \mathrm{g} / \mathrm{mL})$. *In 2009-2011, the 1 ceftriaxone-nonsusceptible isolate was serotype 14 . $\dagger$ In 2011-2013, the 5 ceftriaxonenonsusceptible isolates included 2 of serotype $19 \mathrm{~F}$ and 1 each of serotypes 19A, 34, and 23B. łln 2013-2015, the ceftriaxonenonsusceptible isolates were serotypes $23 F(n=2)$ and $19 A(n=$ $3)$, and 1 was not typeable.
Table 3. Multivariate logistic model for predictors of death among IPD patients, Israel, July 1, 2009-June 30, 2015*

\begin{tabular}{|c|c|c|}
\hline Variable & $\begin{array}{c}\text { Adjusted OR } \\
(95 \% \mathrm{Cl}) \\
\end{array}$ & $\begin{array}{c}\text { Adjusted } \\
\text { p value }\end{array}$ \\
\hline \multicolumn{3}{|l|}{ Age, y } \\
\hline $18-49$ & & Referent \\
\hline $50-64$ & $2.064(1.39-3.06)$ & 0.0003 \\
\hline$\geq 65$ & $4.847(3.42-6.87)$ & $<0.0001$ \\
\hline \multicolumn{3}{|l|}{ Risk group } \\
\hline Not at risk & & Referent \\
\hline At risk & $1.338(0.99-1.80)$ & 0.0561 \\
\hline High risk & $1.609(1.23-2.11)$ & 0.0006 \\
\hline \multicolumn{3}{|l|}{ Serotype by VT } \\
\hline VT7 & & Referent \\
\hline VT13-7 & $0.903(0.65-1.25)$ & 0.539 \\
\hline Non-VT13 & $1.119(0.84-1.50)$ & 0.449 \\
\hline Nonmeningitis IPD & & Referent \\
\hline Pneumococcal meningitis & $0.747(0.50-1.11)$ & 0.153 \\
\hline${ }^{*} \mid \mathrm{PD}$, invasive pneumococcal & OR, odds ratio; $\mathrm{VT}$, va & ine type. \\
\hline
\end{tabular}

that year. The diversity of the serotypes causing pneumococcal meningitis did not change substantially over the 6-year study; the Simpson diversity index was 0.951-0.974. To assess the dynamics of the serotypes commonly causing pneumococcal meningitis, we compared the serotype distribution of the first 2 study years (July 1, 2009-June 30, 2011; pre-PCV13 period) with that of the last 2 study years (July 1, 2013-June 30, 2015) (Figure 4). The dynamics of PCV13 serotype 3 contrasted with those of the other PCV13 serotypes; while the proportion and incidence of the common VT13 serotypes 6A, 23F, 19A, and 7F decreased, serotype 3 slightly increased. Despite the significant decrease in the incidence of VT13 strains and the elimination of VT7 strains, the overall incidence of pneumococcal meningitis increased because of a significant emergence of non-VT13 serotypes (IRR $2.352,95 \%$ CI $1.27-4.35$ ).

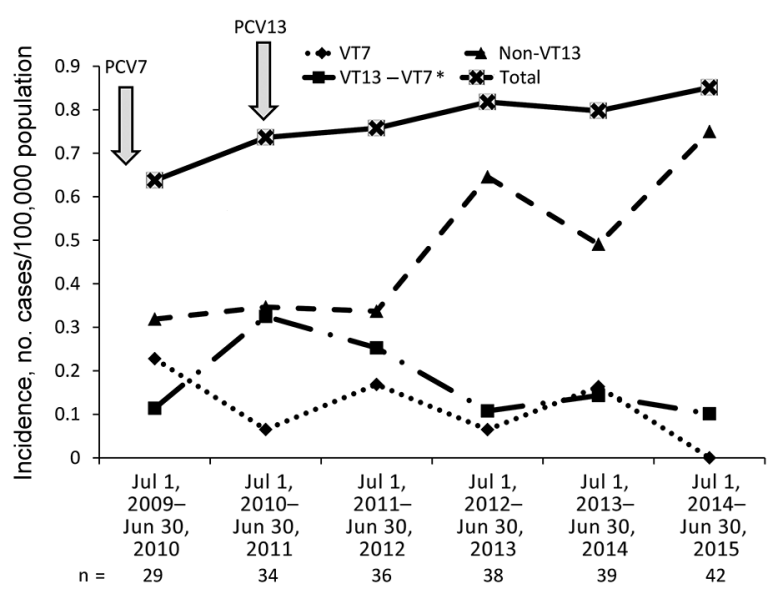

Figure 3. Incidence of pneumococcal meningitis in patients $\geq 18$ years of age, by VT, Israel, July 1, 2009-June 30, 2015. The total number of cases per year are shown, and the introductions of PCV7 and PCV13 into the pediatric national immunization plan are depicted with arrows. *Serotypes included in the VT13 vaccine but not in the VT7 vaccine. PCV, pneumococcal conjugate vaccine; VT, vaccine type. 


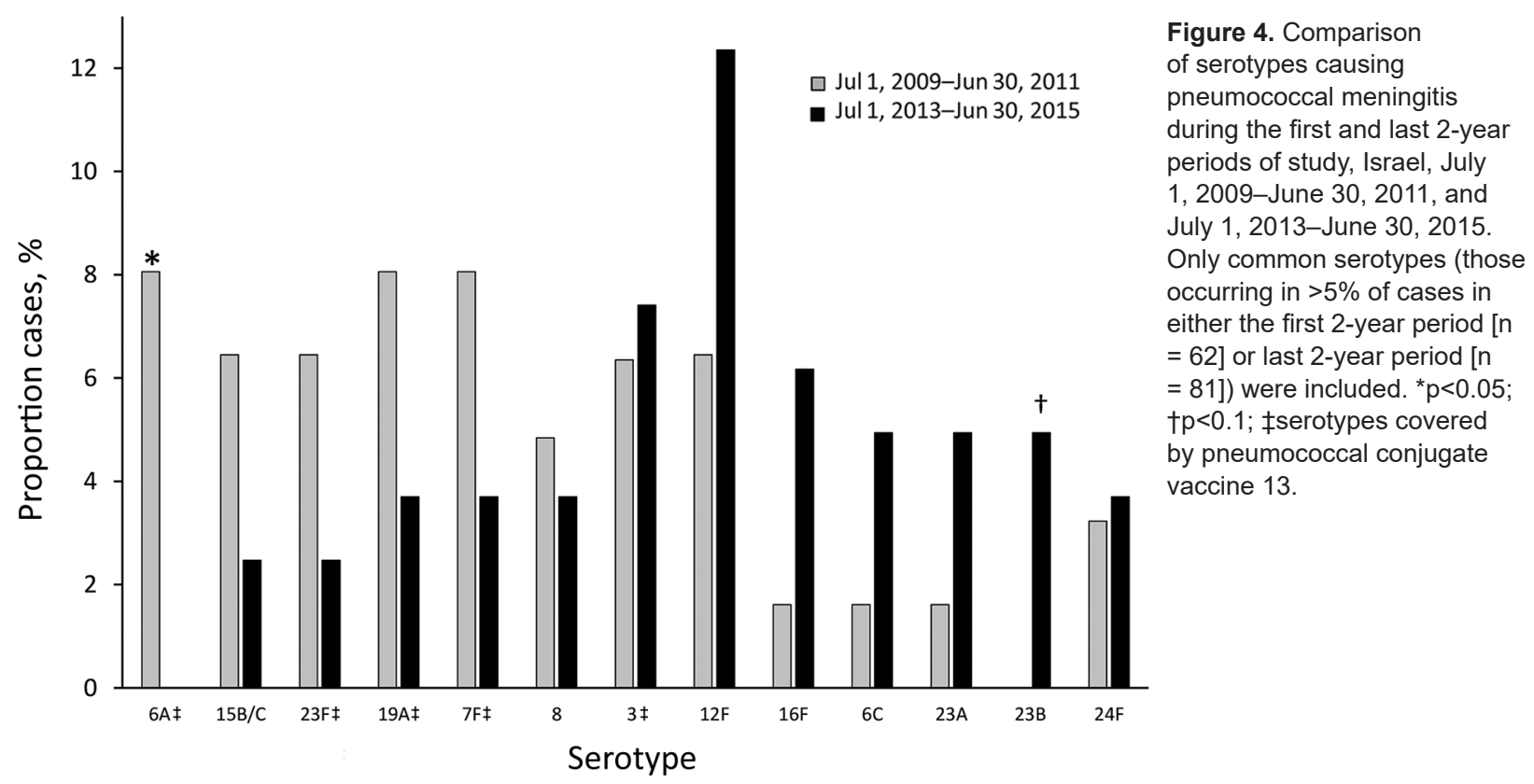

The common emerging non-VT serotypes were $12 \mathrm{~F}, 16 \mathrm{~F}$, $6 \mathrm{C}, 23 \mathrm{~A}, 23 \mathrm{~B}$, and 24F.

Certain serotypes made up a significantly higher proportion of meningitis IPD than nonmeningitis IPD cases, particularly 23A $(\mathrm{p}=0.0018)$ and $23 \mathrm{~B}(\mathrm{p}=0.0001)$ but also $24 \mathrm{~F}(\mathrm{p}=0.054), 23 \mathrm{~F}(\mathrm{p}=0.072), 15 \mathrm{~B} / \mathrm{C}(\mathrm{p}=0.035)$, and $6 \mathrm{C}(\mathrm{p}=0.084)$ (all but $23 \mathrm{~F}$ being non-VT13 serotypes) (Figure 5; online Technical Appendix Table 2). Other serotypes were commonly isolated from patients with nonmeningitis IPD and rarely detected in patients with meningitis IPD, particularly serotypes $14(\mathrm{p}=0.093), 1(\mathrm{p}=0.0002)$, $5(\mathrm{p}=0.0013)$, and $9 \mathrm{~V}(\mathrm{p}=0.045)$.

\section{Predictors of Pneumococcal Meningitis}

To elucidate unrecognized risks or predictors for pneumococcal meningitis beyond neurosurgery or CSF leak, we separately assessed all patients with IPD categorized as not at risk for IPD (i.e., without any recognized concurrent medical condition for which PPSV23 is recommended). First, we performed a univariate analysis comparing characteristics of the not at-risk patients with pneumococcal meningitis with those of the not at-risk patients with nonmeningitis IPD (online Technical Appendix Table 3). The medical conditions trauma, lipid disorders, and chronic or recurrent infections were more frequently reported for the meningitis IPD not at-risk population than the nonmeningitis IPD not at-risk population. Smoking and dementia were less frequently reported for the meningitis IPD patient population. To determine independent predictors for pneumococcal meningitis among the not at-risk patient population, we performed a multivariate logistic analysis. In general, having any concurrent medical condition was associated with nonmeningitis IPD, and the particular medical conditions lipid disorder, chronic or recurrent infections, and previous trauma were independently associated with meningitis IPD. Many of the chronic or recurrent infections reported included otitis media or sinusitis, but the numbers of these cases were too small for greater resolution (online Technical Appendix Table 3). Because we observed that certain serotypes were particularly associated with pneumococcal meningitis, we tested several models to determine whether particular serotypes predicted meningitis IPD in the not at-risk group. The best-fit model found that serogroup 23 was an independent predictor of pneumococcal meningitis in this patient population; adjusted odds ratio was 5.43 (95\% CI 2.01-14.70) (Table 4).

\section{Discussion}

In our nationwide study, we assessed the dynamics of the incidence of pneumococcal meningitis in adults after sequential implementation of PCV7 and PCV13 in children. Our data demonstrate that some features of pneumococcal meningitis differ significantly from those of nonmeningitis IPD. The pneumococcal meningitis patient population was younger and had less frequent and different concurrent medical conditions. Outcomes also differed, with meningitis IPD patients having a more frequent need for ventilation, intensive care treatment, and treatment in long-term care facilities after discharge and a longer length of hospital stay. Yet mortality rates and case-fatality rates did not differ between the meningitis and nonmeningitis IPD populations. Although this finding seems surprising, a decrease in the pneumococcal meningitis case-fatality rate with adjuvant corticosteroid treatment was reported in a multi- 


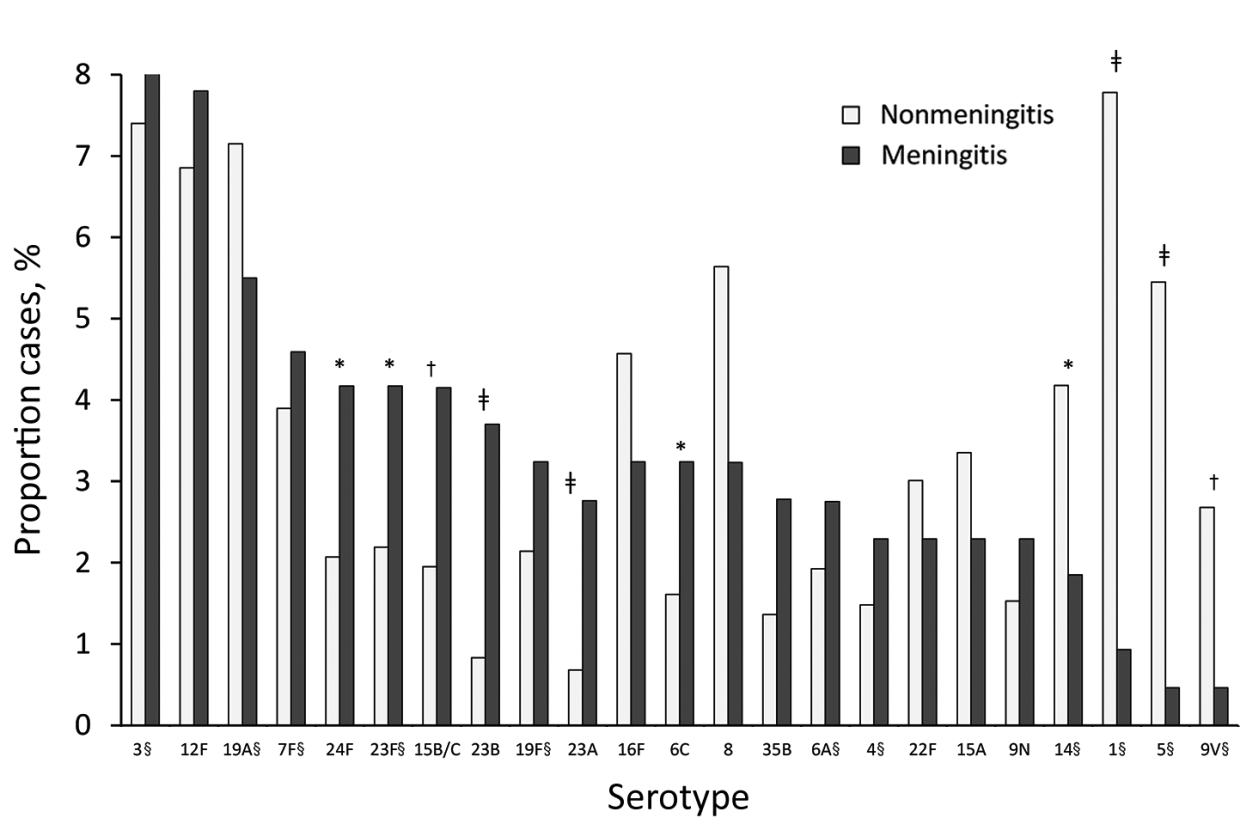

Figure 5. Serotypes associated with meningitis and nonmeningitis invasive pneumococcal disease, Israel, July 1, 2009-June 30, 2015 . Only major serotypes (those totaling $>3 \%$ of all Streptococcus pneumoniae isolates from all study years) were included. ${ }^{*} p<0.1$; $\dagger p<0.05 ; \ddagger p<0.005 ;$ §serotypes covered by pneumococcal conjugate vaccine 13 .

center study in Europe (21). Although we do not know the proportion of patients in our study treated with corticosteroids, this treatment is part of routine practice for treating meningitis patients in Israel.

Ceftriaxone nonsusceptibility increased during the study period. This finding was unexpected because the only nonsusceptible isolate in the pre-PCV13 era was VT7 serotype 14. Antimicrobial drug recommendations did not change in a way that could have stimulated the emergence of ceftriaxone-nonsusceptible isolates. The emerging ceftriaxone-nonsusceptible isolates belonged mainly to serogroups 19 and 23 or non-VT13 serotypes (34 and an unencapsulated isolate [nontypeable]). Previous studies reported initial declines in rates of IPD with antimicrobial drug-resistant $S$. pneumoniae after the introduction of PCV7 in children and adults. These declines were attributed to the reduction of VT7 serotypes, which constituted most of the resistant strains. This observation of increased antimicrobial drug resistance is particularly worrisome; with ceftriaxone-nonsusceptible isolates totaling $8.3 \%$ of meningitis IPD cases, adding vancomycin to first-line therapy (pending culture results) might be necessary. Most of the isolates not susceptible to ceftriaxone were from serotypes covered by PCV13; thus, the ceftriaxone-nonsusceptible VT13 strains might be eventually eliminated through vaccination efforts, and these high nonsusceptibility rates might eventually decline with time. Although the European Society of Clinical Microbiology and Infectious Diseases and the Infectious Diseases Society of America guidelines $(22,23)$ suggest adding vancomycin if a high prevalence of nonsusceptibility to penicillins and cephalosporins is observed, the guidelines do not specify at which resistance prevalence the policy should be enacted. Our results indicate the need for continued surveillance of ceftriaxone nonsusceptibility rates among pneumococcal meningitis cases.

The effect of the PCV NIP for children on pneumococcal meningitis in adults has been studied infrequently and mostly in the pre-PCV13 era. A review summarizing the effect of PCV7 on pneumococcal meningitis incidence in Europe and the United States reported a large variation in the effects, ranging from a $137 \%$ increase to a $77 \%$ decrease; the effect on VT7 pneumococcal meningitis incidence was also diverse (ranging from a $43 \%$ increase to an $87 \%$ decrease) (24). Studies in the United States and the Netherlands reported reductions in overall pneumococcal meningitis in adults after PCV7 or PCV10 introduction $(13,14)$. Data on the effects of PCV13 on pneumococcal meningitis are scarce and include only data on pediatric populations; results differed according to geographic region. No significant change in pediatric pneumococcal meningitis rates was reported in 2 studies $(9,10)$, and a decrease in pneumococcal meningitis in children $<2$ years of age was reported in others $(22,25)$.

We show that in contrast to the significant indirect effect observed on overall IPD and particularly bacteremic pneumococcal pneumonia $(4,26,27)$, adult pneumococcal meningitis incidence did not decrease after PCV implementation. We show that the reason for this is an increase of pneumococcal meningitis incidence caused by non-VT13 serotypes. A significant replacement in carriage of less invasive non-VT13 serotypes in children occurred after the PCV13 introduction; these strains had higher rates of 
Table 4. Multivariate logistic model for predictors of meningitis among patients not at risk for IPD, Israel, July 1, 2009-June 30, $2015^{\star}$

\begin{tabular}{|c|c|c|}
\hline Variable & $\begin{array}{l}\text { Adjusted OR } \\
(95 \% \mathrm{Cl})\end{array}$ & $\begin{array}{l}\text { Adjusted } \\
p \text { value }\end{array}$ \\
\hline \multicolumn{3}{|l|}{ Age, y } \\
\hline $18-49$ & & Referent \\
\hline $50-64$ & $2.13(1.16-3.91)$ & 0.015 \\
\hline$\geq 65$ & & 0.042 \\
\hline \multicolumn{3}{|l|}{ Concurrent medical conditions } \\
\hline None & & Referent \\
\hline Any† & $0.48(0.27-0.85)$ & 0.011 \\
\hline No history of previous trauma & & Referent \\
\hline Previous trauma & $9.78(2.24-42.75)$ & 0.002 \\
\hline No lipid disorder & & Referent \\
\hline Lipid disorder & $2.73(1.19-6.24)$ & 0.017 \\
\hline No history of infections & & Referent \\
\hline $\begin{array}{l}\text { Recurrent or chronic } \\
\text { infections } \ddagger\end{array}$ & $12.31(3.86-39.22)$ & $<0.0001$ \\
\hline No history of dementia & & Referent \\
\hline Dementia & $0.12(0.02-0.91)$ & 0.040 \\
\hline \multicolumn{3}{|l|}{ Serotype } \\
\hline Non-23 & & Referent \\
\hline 23 & $5.43(2.01-14.70)$ & 0.0009 \\
\hline \multicolumn{3}{|c|}{$\begin{array}{l}\text { *Patients not at risk for IPD were defined as those without any recognized } \\
\text { concurrent medical condition for which PPSV23 administration is } \\
\text { recommended. IPD, invasive pneumococcal disease; OR, odds ratio; } \\
\text { PPSV23, pneumococcal polysaccharide vaccine } 23 \text {. } \\
\text { †Any concurrent medical condition, excluding those for which PPSV23 is } \\
\text { recommended. Patients with these conditions were not included in the not } \\
\text { at-risk group. } \\
\text { IVarious types of recurrent or chronic infections, including chronic otitis } \\
\text { media, sinusitis, recurrent cellulitis, chronic osteomyelitis, and } \\
\text { tuberculosis. }\end{array}$} \\
\hline
\end{tabular}

transmission to adults (7). But why these non-VT serotypes cause more meningitis than nonmeningitis IPD is unclear. Our data suggest roles of both the host and the pathogen; a higher proportion of patients with meningitis IPD had previous neurosurgery operations, CSF leak, and history of trauma, during which direct penetration of carried bacteria could potentially occur and explain the greater replacement with non-PCV13 serotypes. We also found that odds of pneumococcal meningitis development was higher among patients with a lipid disorder and lower among patients with dementia. We have no explanations for these findings. The latter observation could potentially be explained by classification bias, if less diagnostic efforts were taken to understand the status of patients with dementia.

We also show that independent of concurrent medical conditions, several serotypes, mostly non-VT13 serotypes, were significantly associated with meningitis, suggesting a specific role of the pathogen in patients with concurrent medical conditions. Serotypes $6 \mathrm{C}$ and 23 are either directly covered by PCV13 or indirectly covered through crossprotection from antibodies developed against serotypes $6 \mathrm{~B}$ and $23 \mathrm{~F}$. The only previous study of the indirect effects of PCV13 childhood vaccination on adult pneumococcal meningitis reported similar results as ours; however, these results were based on a small sample size $(<20$ cases of pneumococcal meningitis) (16).
This study has several limitations. First, data from the pre-PCV7 era were not collected. Second, antimicrobial drug susceptibility testing was performed in different laboratories and variations in testing might have occurred. Third, meningitis cases that were diagnosed on the basis of PCR without culturing were not included, and thus, meningitis rates might be underestimated. Last, this study has the limitations of a retrospective study; thus, only data available from the electronic file were collected.

In conclusion, this nationwide study indicates that PCV7 and PCV13 childhood vaccination indirectly affected the incidence of adult pneumococcal meningitis. We report the elimination of the occurrence of VT7 serotypes among pneumococcal meningitis patients within 6 years after PCV7 implementation and 4.5 years after PCV13 implementation. Yet we report no decrease in overall IPD meningitis cases because of the significant emergence of several non-VT13 strains, particularly serotypes $6 \mathrm{C}, 12 \mathrm{~F}$, $16 \mathrm{~F}, 23 \mathrm{~A}$, and 23B.

The IAIPD study principal investigator was Gili Regev-Yochay and the IsraNIP Project principal investigator was Ron Dagan.

Additional members of the IAIPD group who contributed: Marc Assous (Shaare Zedek Medical Center, Jerusalem, Israel); Haim Ben-Zvi, Jihad Bishara (Rabin Medical Center, Petah Tikva, Israel); Rita Bardenstein, Oren Zimchony (Kaplan Medical Center, Rehovot, Israel); Larissa Brik, Miriam Weinberger (Assaf Harofeh Medical Center, Tzrifin, Israel); Bibiana Chazan, Yoram Kennes (HaEmek Medical Center, Afula, Israel); Michal Chowers, Yosi Paitan (Meir Medical Center, Kfar Saba, Israel); Ronit Cohen-Poradosu, Talia Finn, Yael Paran, David Schwartz (Tel Aviv Medical Center, Tel Aviv, Israel); Alicia Embon (Barzilai Medical Center, Ashkelon, Israel); Sarit Freimann, (Hillell Yaffe Medical Center, Hadera, Israel); Yuval Geffen, Ilana Oren (Rambam Medical Center, Haifa, Israel); Danny Glikman, Shifra Sela (Western Galilee Hospital, Nahariya, Israel); Mirit Hershman, Israel Potasman, Itzhak Srugo (Bnai Zion Medical Center, Haifa); Gill Smollan (Sheba Medical Center, Ramat Gan, Israel); Camellia Khoury-Assi (French Hospital, Nazareth, Israel); Mandelbaum Sari (Laniado Hospital - Sanz Medical Center, Netanya, Israel); Yasmin Maor, Orna Schwartz, Michal Stein (Edith Wolfson Medical Center, Holon, Israel); Danny Miron, Hagai Rechnitzer (Ziv Medical Center, Safed, Israel); Nehama Peled, Nurit Porat, (Soroka University Medical Center, Beersheba, Israel); Pninit Shaked-Mishan, Gabriel Weber (Carmel Medical Center, Haifa); Yehudit Sheindler (Maayanei Hayeshua Hospital, Bnei Brak, Israel); Violetta Temper (Hadassah-Hebrew University, Jerusalem); Olga Sverdlob (Maccabi Healthcare Services, Rehovot).

\section{Acknowledgments}

We thank Ronit Trefler for serotyping and Efrat Steinberger and Etti Kreiff for their assistance in data collection and management. 
The IAIPD is part of the IsraNIP project. This study was supported in part by Wyeth (Pfizer), manufacturer of Prevnar 7 and Prevnar 13 (grant no. 0887Z1-4603). Wyeth (Pfizer) had no role in designing the study, collecting the data, analyzing the data, interpreting the data, writing the report, or in the decision to submit the paper for publication.

G.R.Y. has served as a consultant for Neopharm and Pfizer. G.R. has served as a consultant for MSD, AstraZeneca, Pfizer, and Astellas. R.D. has received grants/research support from Berna/Crucell, Wyeth/Pfizer, MSD, and Protea; has been a scientific consultant for Berna/Crucell, Glaxo-SmithKline, Novartis, Wyeth/Pfizer, Protea, and MSD; has been a speaker for Berna/Crucell, GlaxoSmithKline, and Wyeth/Pfizer; and is a shareholder of Protea/NASVAX.

\section{About the Author}

Dr. Regev-Yochay is an infectious disease specialist and an infectious disease epidemiologist serving as the director of the Infection Control and Prevention Unit at the Sheba Medical Center and the head of the Infectious Disease Epidemiology Section at the Gertner Research Institute. Much of her research is focused on pneumococcal infections, carriage, and the direct and indirect effects of vaccination.

\section{References}

1. Thigpen MC, Whitney CG, Messonnier NE, Zell ER, Lynfield R, Hadler JL, et al.; Emerging Infections Programs Network. Bacterial meningitis in the United States, 1998-2007. N Engl J Med. 2011;364:2016-25. http://dx.doi.org/10.1056/ NEJMoa1005384

2. Schuchat A, Robinson K, Wenger JD, Harrison LH, Farley M, Reingold AL, et al.; Active Surveillance Team. Bacterial meningitis in the United States in 1995. N Engl J Med. 1997;337:970-6. http://dx.doi.org/10.1056/NEJM199710023371404

3. Pilishvili T, Lexau C, Farley MM, Hadler J, Harrison LH, Bennett NM, et al.; Active Bacterial Core Surveillance/ Emerging Infections Program Network. Sustained reductions in invasive pneumococcal disease in the era of conjugate vaccine. J Infect Dis. 2010;201:32-41. http://dx.doi.org/10.1086/ 648593

4. Regev-Yochay G, Paran Y, Bishara J, Oren I, Chowers M, Tziba Y, et al.; Israeli Adult Invasive Pneumococcal Disease group. Early impact of PCV7/PCV13 sequential introduction to the national pediatric immunization plan, on adult invasive pneumococcal disease: a nationwide surveillance study. Vaccine. 2015;33:113542. http://dx.doi.org/10.1016/j.vaccine.2015.01.030

5. Waight PA, Andrews NJ, Ladhani NJ, Sheppard CL, Slack MP, Miller E. Effect of the 13-valent pneumococcal conjugate vaccine on invasive pneumococcal disease in England and Wales 4 years after its introduction: an observational cohort study. Lancet Infect Dis. 2015;15:535-43. http://dx.doi.org/10.1016/ S1473-3099(15)70044-7

6. Moore MR, Link-Gelles R, Schaffner W, Lynfield R, Lexau C, Bennett NM, et al. Effect of use of 13-valent pneumococcal conjugate vaccine in children on invasive pneumococcal disease in children and adults in the USA: analysis of multisite, populationbased surveillance. Lancet Infect Dis. 2015;15:301-9. http://dx.doi.org/10.1016/S1473-3099(14)71081-3
7. Weinberger DM, Trzciński K, Lu YJ, Bogaert D, Brandes A, Galagan J, et al. Pneumococcal capsular polysaccharide structure predicts serotype prevalence. PLoS Pathog. 2009;5:e1000476. http://dx.doi.org/10.1371/journal.ppat.1000476

8. Chowers M, Regev-Yochay G, Mor O, Cohen-Poradosu R, Riesenberg K, Zimhony O, et al. Invasive pneumococcal disease (IPD) in HIV infected patients in Israel since the introduction of pneumococcal conjugated vaccines (PCV): analysis of a nationwide surveillance study, 2009-2014. Hum Vaccin Immunother. 2017;13:216-9. http://dx.doi.org/10.1080/ 21645515.2016.1229720

9. Olarte L, Barson WJ, Barson RM, Lin PL, Romero JR, Tan TQ, et al. Impact of the 13-valent pneumococcal conjugate vaccine on pneumococcal meningitis in US children. Clin Infect Dis. 2015;61:767-75. http://dx.doi.org/10.1093/cid/civ368

10. Barrett C, Ben-Shimol S, Greenberg D. Differences between radiologically confirmed pneumonia with and without pleural fluid in hospitalized children younger than 5 years in southern Israel. Clin Pediatr (Phila). 2016;55:897-903. http://dx.doi.org/ $10.1177 / 0009922815616246$

11. Bielecka MK, Tezera LB, Zmijan R, Drobniewski F, Zhang X, Jayasinghe $\mathrm{S}$, et al. A bioengineered three-dimensional cell culture platform integrated with microfluidics to address antimicrobial resistance in tuberculosis. MBio. 2017;8:e2073-16. http://dx.doi.org/10.1128/mBio.02073-16

12. Hsu HE, Shutt KA, Moore MR, Beall BW, Bennett NM, Craig AS, et al. Effect of pneumococcal conjugate vaccine on pneumococcal meningitis. N Engl J Med. 2009;360:244-56. http://dx.doi.org/10.1056/NEJMoa0800836

13. Castelblanco RL, Lee M, Hasbun R. Epidemiology of bacterial meningitis in the USA from 1997 to 2010: a population-based observational study. Lancet Infect Dis. 2014;14:813-9. http://dx.doi.org/10.1016/S1473-3099(14)70805-9

14. Bijlsma MW, Brouwer MC, Kasanmoentalib ES, Kloek AT, Lucas MJ, Tanck MW, et al. Community-acquired bacterial meningitis in adults in the Netherlands, 2006-14: a prospective cohort study. Lancet Infect Dis. 2016;16:339-47. http://dx.doi.org/ 10.1016/S1473-3099(15)00430-2

15. Alari A, Chaussade H, Domenech De Cellès M, Le Fouler L, Varon E, Opatowski L, et al. Impact of pneumococcal conjugate vaccines on pneumococcal meningitis cases in France between 2001 and 2014: a time series analysis. BMC Med. 2016;14:211. http://dx.doi.org/10.1186/s12916-016-0755-7

16. Kendall BA, Dascomb KK, Mehta RR, Stockmann C, Mason EO, Ampofo K, et al. Early Streptococcus pneumoniae serotype changes in Utah adults after the introduction of PCV13 in children. Vaccine. 2016;34:474-8. http://dx.doi.org/10.1016/ j.vaccine.2015.12.010

17. Ben-Shimol S, Givon-Lavi N, Greenberg D, Dagan R. Pneumococcal nasopharyngeal carriage in children $<5$ years of age visiting the pediatric emergency room in relation to PCV7 and PCV13 introduction in southern Israel. Hum Vaccin Immunother. 2016;12:268-76. http://dx.doi.org/10.1080/ 21645515.2015.1095414

18. Ben-Shimol S, Greenberg D, Givon-Lavi N, Elias N, Glikman D, Rubinstein U, et al.; Israeli Bacteremia and Meningitis Active Surveillance Group. Rapid reduction in invasive pneumococcal disease after introduction of $\mathrm{PCV} 7$ into the national immunization plan in Israel. Vaccine. 2012;30:6600-7. http://dx.doi.org/10.1016/j.vaccine.2012.08.012

19. Greenland S, Rothman KJ. Measures of occurrence. In: Rothman KJ, Greenland S, Lash TL, editors. Modern epidemiology. 3rd ed. Philadelphia: Lippincott Williams \& Williams; 2008. p. 32-50.

20. Simpson EH. Measurement of diversity. Nature. 1949;163:688. http://dx.doi.org/10.1038/163688a0 
21. de Gans J, van de Beek D; European Dexamethasone in Adulthood Bacterial Meningitis Study Investigators. Dexamethasone in adults with bacterial meningitis. N Engl J Med. 2002;347:1549-56. http://dx.doi.org/10.1056/NEJMoa021334

22. van de Beek D, Cabellos C, Dzupova O, Esposito S, Klein M, Kloek AT, et al.; ESCMID Study Group for Infections of the Brain (ESGIB). ESCMID guideline: diagnosis and treatment of acute bacterial meningitis. Clin Microbiol Infect. 2016;22(Suppl 3):S37-62. http://dx.doi.org/10.1016/j.cmi.2016.01.007

23. Tunkel AR, Hartman BJ, Kaplan SL, Kaufman BA, Roos KL, Scheld WM, et al. Practice guidelines for the management of bacterial meningitis. Clin Infect Dis. 2004;39:1267-84. http://dx.doi.org/10.1086/425368

24. Tin Tin Htar M, Madhava H, Balmer P, Christopoulou D, Menegas D, Bonnet E. A review of the impact of pneumococcal polysaccharide conjugate vaccine (7-valent) on pneumococcal meningitis. Adv Ther. 2013;30:748-62. http://dx.doi.org/10.1007/ s12325-013-0051-2
25. Chapoutot AG, Dessein R, Guilluy O, Lagrée M, Wallet F, Varon E, et al. Impact of the 13-valent pneumococcal conjugate vaccine on the incidence of pneumococcal meningitis in children. Epidemiol Infect. 2016;144:607-11. http://dx.doi.org/10.1017/ S095026881500179X

26. Harboe ZB, Dalby T, Weinberger DM, Benfield T, Mølbak K, Slotved HC, et al. Impact of 13-valent pneumococcal conjugate vaccination in invasive pneumococcal disease incidence and mortality. Clin Infect Dis. 2014;59:1066-73. http://dx.doi.org/10.1093/cid/ciu524

27. Greenberg D, Givon-Lavi N, Ben-Shimol S, Ziv JB, Dagan R. Impact of PCV7/PCV13 introduction on community-acquired alveolar pneumonia in children $<5$ years. Vaccine. 2015;33:4623-9. http://dx.doi.org/10.1016/j.vaccine.2015.06.062

Address for correspondence: Gili Regev-Yochay, Sheba Medical Center, Infectious Disease Unit, Ramat Gan, Israel; email: gili.regev@sheba.health.gov.il

\section{June 2014: Respiratory Infections}

- Adverse Pregnancy Outcomes and Coxiella burnetii Antibodies in Pregnant Women, Denmark

- Novel Henipa-like Virus, Mojiang Paramyxovirus, in Rats, China, 2012

- Genetic Evidence of Importation of Drug-Resistant Plasmodium falciparum to Guatemala from the Democratic Republic of the Congo

- Short-Term Malaria Reduction by Single-Dose Azithromycin during Mass Drug Administration for Trachoma, Tanzania
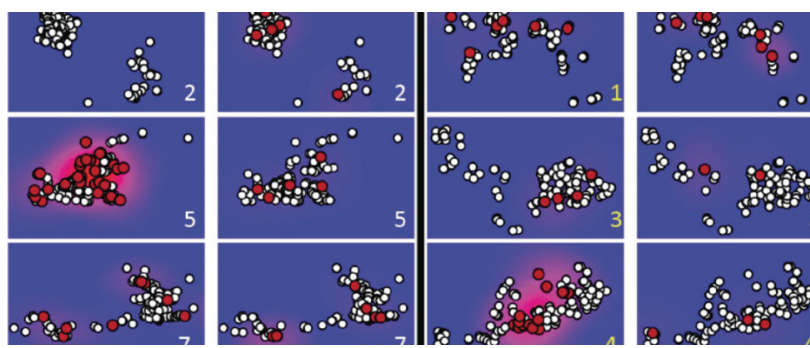

- Rapid Spread and Diversification of Respiratory Syncytial Virus Genotype ON1, Kenya

- Bats as Reservoir Hosts of Human Bacterial Pathogen, Bartonella mayotimonensis

- Oral Fluid Testing for Pertussis, England and Wales, June 2007-August 2009

- High Prevalence of Ancylostoma ceylanicum Hookworm Infections in Humans, Cambodia, 2012

- Characteristics of Patients with Mild to Moderate Primary Pulmonary Coccidioidomycosis

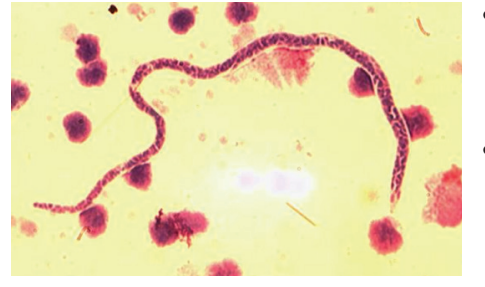

- Human Polyomavirus 9 Infection in Kidney Transplant Patients

- Infection with Mansonella perstans Nematodes in Buruli Ulcer Patients, Ghana

- Timeliness of Yellow Fever Surveillance, Central African Republic

- Gastroenteritis Outbreaks Caused by a DS-1-like G1P[8] Rotavirus Strain, Japan, 2012-2013

- Novel Human Bufavirus Genotype 3 in Children with Severe Diarrhea, Bhutan

- Fatal Monkeypox in Wild-Living Sooty Mangabey, Côte d'Ivoire, 2012

- Human Infection with MERS Coronavirus after Exposure to Infected Camels, Saudi Arabia, 2013

- Sequential Gastroenteritis Episodes Caused by 2 Norovirus Genotypes

- Species H Rotavirus Detected in Piglets with Diarrhea, Brazil, 2012

- latrogenic Meningitis Caused by Neisseria sicca/subflava after Intrathecal Contrast Injection, Australia

- Identification of Possible Virulence Marker from Campylobacter jejuni Isolates

- Novel Phlebovirus with Zoonotic Potential Isolated from Ticks, Australia

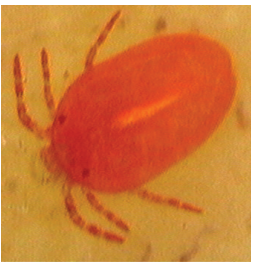

